\title{
A physiotherapy network provided with training, monitoring and support achieves improved return to work and health outcomes
}

\author{
Paul Coburn \\ Victorian WorkCover Authority \& Transport Accident Commission, Melbourne VIC 3000, Australia. paul_coburn@vwa.vic.gov.au
}

Background: In 2007, the Health and Disability Strategy Group (HDSG) of the TAC and Victorian WorkCover Authority recognised an opportunity to improve scheme outcomes by working more closely with health disciplines. To investigate whether this was feasible, a pilot program was proposed with a select group of physiotherapists. Objectives: To determine whether a network of physiotherapists who were provided with training, monitoring and support could achieve improved outcome in return to work and health outcomes.

Method: In 2008, 92 physiotherapists undertook a select tender process and then completed training in return to work, the HDSG's Clinical Framework and familiarisation of the compensation system for work related and motor accident injuries. The outcomes of this network physiotherapist program were evaluated internally and by three independent research organisations to determine 1) impact on return to work, 2) impact on health outcomes, 3) patient satisfaction and 4) satisfaction of the physiotherapist providing the service. Results: When compared to non-network physiotherapist, the network physiotherapists demonstrated improved return to work for clients, better health outcomes, and greater patient satisfaction. Therapist satisfaction was also higher within the network physiotherapists.

Discussion: This body of work conducted on a pilot group of physiotherapists demonstrates improved outcomes in a number of important domains for the management of worker's and motor accident injuries. It indicates that there is a benefit for schemes in supporting clinical programs. Current evidence suggests that compensable patients have worse health outcomes than their non-compensable counterparts. There is a need to promote programs that are beneficial to patients, compensable schemes and providers. Further investigation is required to demonstrate this benefit on a larger scale.

Conclusions: This study indicates that there may be opportunities to improve health outcomes by investment from compensable bodies in training, support and monitoring of specific health disciplines. 